\title{
A pooled analysis of the LAMP assay for the detection of Neisseria meningitidis
}

\author{
Shu-Jin Fan ${ }^{1,2}$, Hong-Kun Tan ${ }^{1,2}$, Yu-Cheng Xu ${ }^{1,2}$, Yuan-Zhi Chen ${ }^{1,2}$, Tian-Ao Xie ${ }^{1,2}$, Zhi-Yong Pan ${ }^{1,2}$, Shi Ouyang ${ }^{3}$, \\ Qin Li ${ }^{4}$, Xiao-yan Li ${ }^{5}$ Zhen-Xing $\mathrm{Li}^{6}$ and Xu-Guang Guo ${ }^{1,2,7,8^{*}}$ (D)
}

\begin{abstract}
Background: Neisseria meningitidis is a major cause of bacterial meningitis, and these infections are associated with a high mortality rate. Rapid and reliable diagnosis of bacterial meningitis is critical in clinical practice. However, this disease often occurs in economically depressed areas, so an inexpensive, easy to use, and accurate technology is needed. We performed a pooled-analysis to assess the potential of the recently developed loop-mediated isothermal amplification (LAMP) assay for detection of meningococcus.

Methods: Pubmed, Embase, and Web of Science were searched to identify original studies that used the LAMP assay to detect meningococcus. After pooling of data, the sensitivity and specificity were calculated, a summary receiver operating characteristic (SROC) curve was determined, and the area under the SROC curve was computed to determine diagnostic accuracy. Publication bias was assessed using Deek's funnel plot.

Results: We examined 14 studies within 6 publications. The LAMP assay had high sensitivity (94\%) and specificity $(100 \%)$ in the detection of meningococcus in all studies. The area under the SROC curve (0.980) indicated high overall accuracy of the LAMP assay. There was no evidence of publication bias.

Discussion: The LAMP assay has accuracy comparable to bacterial culture and PCR for detection of meningococcus, but is less expensive and easier to use. We suggest the adoption of the LAMP assay to detect meningococcus, especially in economically depressed areas.
\end{abstract}

Keywords: Neisseria meningitidis, Meningitis, LAMP assay, Pooled-analysis

\section{Background}

Neisseria meningitidis is a Gram-negative diplococci bacterium that only infects humans, and is a significant cause of meningitis. Neisseria meningitidis parasitizes in patient's cerebrospinal fluid easily in the acute and early stage of diseases. But it is unknown of the carriage rate in healthy individuals at presen $t[1]$. Based on capsular polysaccharides, there are 13 serogroups of this species (A, B, C, D, E, H, I, K, L, W, X, Y, and Z ) [2] Serogroups A, B, C,

\footnotetext{
* Correspondence: gysygxg@gmail.com

'Department of Clinical Laboratory Medicine, the Third Affiliated Hospital of Guangzhou Medical University, Guangzhou 510150, China

${ }^{2}$ Department of Clinical Medicine, the Third Clinical School of Guangzhou Medical University, Guangzhou 511436, China

Full list of author information is available at the end of the article
}

Y, and W-135 are mainly responsible for human diseases, [3] and serogroups $A$ and $C$ are mainly responsible for meningitis. By the way we found other groups such as group B, Y and W135 cause severe epidemic lots of lands. The incidence of bacterial meningitis is greatest among infants under 1 year-old, followed by adolescent $s[4]$.

The overall incidence of meningitis has declined in recent decades due to the increasing use of meningococcal vaccines, [5] but the incidence of meningitis from bacterial strains not covered by vaccines has increased, and bacterial meningitis remains a significant public health problem. About 200,000 patients worldwide die from bacterial meningitis every year, and the mortality can be up to $60 \%$ in parts of sub-Saharan Africa and in poor

(c) The Author(s). 2020, corrected publication 2020. Open Access This article is licensed under a Creative Commons Attribution 4.0 International License, which permits use, sharing, adaptation, distribution and reproduction in any medium or format, as long as you give appropriate credit to the original author(s) and the source, provide a link to the Creative Commons licence, and indicate if changes were made. The images or other third party material in this article are included in the article's Creative Commons licence, unless indicated otherwise in a credit line to the material. If material is not included in the article's Creative Commons licence and your intended use is not permitted by statutory regulation or exceeds the permitted use, you will need to obtain permission directly from the copyright holder. To view a copy of this licence, visit http://creativecommons.org/ licenses/by/4.0/. The Creative Commons Public Domain Dedication waiver (http://creativecommons.org/publicdomain/zero/1. 0/) applies to the data made available in this article, unless otherwise stated in a credit line to the data. 
and developing countrie s[6,7]. Patients who receive treatment have a mortality rate of about $10 \%$, but survivors often experience serious sequelae, including limb amputation, neurological deficits, and other serious disabilitie s[8].

Clinical detection of Neisseria meningitidis in many countries currently uses PCR, ELISA, and bacterial culture,[9] and PCR is considered the most authoritative standard. However, these technologies have limited applicability in poor and developing countries. For example, PCR and ELISA can be time-consuming, difficult to perform, and relatively expensiv e[10]. Identification by bacterial culture can take many days, has low sensitivity, and has a reduced detection rate for patients who were pretreated with antibiotic s[6].

Loop-mediated isothermal amplification (LAMP) is a relatively new nucleic acid amplification technology that can simultaneously detect six meningococcal groups of $N$. meningitidis (A, B, C, W, X, and Y). It is simple to perform, rapid, has high sensitivity, and is inexpensive, [11] making it especially suitable for developing countries and regions with limited resources. No previous studies have comprehensively analyzed the sensitivity and specificity of the LAMP assay for detection of Neisseria meningitidis. The present analysis evaluated the diagnostic accuracy and feasibility of using LAMP to identify meningitis due to Neisseria meningitidis. This analysis also included the assessment of publication bias and data quality.

\section{Methods}

\section{Study design}

A systematic review of the diagnostic accuracy of LAMP in Neisseria meningitidis was performed, followed by a pooled-analysis.

\section{Search strategy and study selection}

The phrases "Neisseria meningitidis", "Meningococcus", "LAMP" and "Loop-mediated isothermal amplification" were used in combination to systemically search the literature from January 1997 to February 7, 2019 in 5 databases (Pubmed, Embase, Medline, Web of Science, and Cochrane Library) for identification of original studies that used LAMP to detect Neisseria meningitidis. The bibliographies of all publications were also reviewed to identify additional studies.

All literature titles and abstracts were independently screened, and five researchers (including Fan Shujin and Tan Hongkun) read the full text of each article that had extractable data to determine its eligibility for inclusion. Differences regarding eligibility were resolved through negotiation. Each included study used LAMP for detection of Neisseria meningitidis and a gold standard test (PCR or culture); provided data that were extractable and included true positivity (TP), false positivity (FP), false negativity (FN), and true negativity (TN) of the LAMP assay; and was original research written in English or Chinese. Studies that were animal experiments, from conferences or literature reviews, or did not have extractable data were excluded.

\section{Data extraction and quality assessment}

Five researchers independently extracted the data from each included study, including author, publication year, sample size, sample type, gold standard test results, TP, FP, FN, and TN. Regarding patient selection, index test, reference standard, flow and timing were used to evaluate the diagnostic accuracy of the LAMP assay.

\section{Statistical analysis}

MetaDisc version 1.4.0.0 was used to calculate sensitivity, specificity, positive likelihood ratio (PLR), negative likelihood ratio (NLR), and diagnostic odds ratio (DOR). Diagnostic odds ratio was used as the main outcome measure of the SROC approach of the standard method for analyzing diagnostic studies reporting pairs of sensitivity and specificity. A summary receiver operating characteristic (SROC) curve was plotted for calculations of sensitivity and specificity. A random effects model was used to summarize all data. Deek's funnel plot was drawn using STATA, and a bivariate box plot and a post-test probability plot were used to detect literature bias. Review Manager version 5.2 was used for plotting study quality evaluation.

\section{Results}

\section{Search results and study characteristics}

Our initial literature search identified 83 publications and no additional (gray) literature (Fig. S1). After application of the inclusion and exclusion criteria, we identified 6 publications for inclusion in the analysis, all of which were published between 2011 and 2018 (Table 1). These studies were performed in Ireland, the United Kingdom, South Korea, and Japan. Because some of these publications used multiple types of sampling, we categorized these 6 publications as 14 studies with 3185 samples.

\section{Diagnostic accuracy of LAMP assay}

We used a random effects model to determine the diagnostic accuracy of the LAMP assay. Overall, the sensitivity was $0.94\left(95 \% \mathrm{CI}=0.89\right.$ to $0.98, \mathrm{I}^{2}=24.7 \%$; Fig. 1$)$, the specificity was $1.00\left(95 \% \mathrm{CI}=0.99\right.$ to $1.00, \mathrm{I}^{2}=58 \%$; Fig. 2), the PLR was 69.45 (95\% CI $=30.65$ to 157.37 , $\mathrm{I}^{2}=66.8 \%$; Fig. 3), the NLR was $0.13(95 \% \mathrm{CI}=0.08$ to 0.21 , and $\mathrm{I}^{2}=0.0 \%$; Fig. 4), the DOR was $929.06(95 \%$ $\mathrm{CI}=339.22$ to $2544.53, \mathrm{I}^{2}=18.9 \%$; Fig. 5 ), and SROC 
Table 1 Characteristics of the included studies

\begin{tabular}{|c|c|c|c|c|c|c|c|c|c|}
\hline Author & Year & Country & Patients (n) & Specimen & Referencestandard & TP & FP & FN & $\mathrm{TN}$ \\
\hline McKenna et al .[12] & 2011 & UK & 139 & Throat swab & PCR & 12 & 1 & 0 & 126 \\
\hline McKenna et al .[12] & 2011 & UK & 141 & Serum & PCR & 14 & 1 & 0 & 126 \\
\hline McKenna et al .[12] & 2011 & UK & 72 & Blood & $P C R$ & 4 & 1 & 0 & 67 \\
\hline McKenna et al .[12] & 2011 & UK & 22 & CSF & PCR & 3 & 1 & 0 & 18 \\
\hline McKenna et al .[12] & 2011 & UK & 16 & Respiratory secretions & $P C R$ & 2 & 0 & 0 & 14 \\
\hline McKenna et al .[12] & 2011 & UK & 4 & Feces & PCR & 1 & 0 & 0 & 3 \\
\hline Bourke et al .[13] & 2015 & U.K. & 141 & Nasopharyngeal & PCR & 21 & 0 & 4 & 116 \\
\hline Bourke et al .[13] & 2015 & U.K. & 144 & Blood & PCR & 22 & 0 & 4 & 118 \\
\hline Lee et al. .[14] & 2015 & South Korea & 31 & CSF & Culture & 2 & 3 & 0 & 26 \\
\hline Lee et al .[15] & 2015 & South Korea & 568 & CSF & PCR & 15 & 5 & 0 & 548 \\
\hline Lee et al .[15] & 2015 & South Korea & 536 & CSF & PCR & 5 & 0 & 0 & 531 \\
\hline Lee et al .[15] & 2015 & South Korea & 470 & CSF & PCR & 5 & 1 & 0 & 464 \\
\hline Takahashi et al .[16] & 2016 & Japan & 836 & Throat swab & $P C R$ & 6 & 1 & 0 & 829 \\
\hline Higgins et al .[17] & 2018 & UK & 65 & Blood & PCR & 22 & 0 & 0 & 43 \\
\hline
\end{tabular}

CSF cerebrospinal fluid, $F N$ false negative, $F P$ false positive, $T N$ true negative, $T P$ true positive

analysis indicated the Area Under Curve (AUC) was 0.9805 (Fig. 6).

\section{Quality evaluation}

We assessed the quality of the studies by analysis of patient selection, the index test, the reference standard, and study flow and timing (Figs. 7 and 8). The results indicated that patient selection had a high risk of bias in 3 studies, an unclear risk of bias in 3 studies, and a low risk of bias in 8 studies. Analysis of patient flow and timing indicated that only 1 study had a high risk of bias and 2 studies had an unclear risk of bias. Analysis of the index test indicated an unclear risk of bias in 1 study, and low risk of bias in the other 13 studies. Analysis of

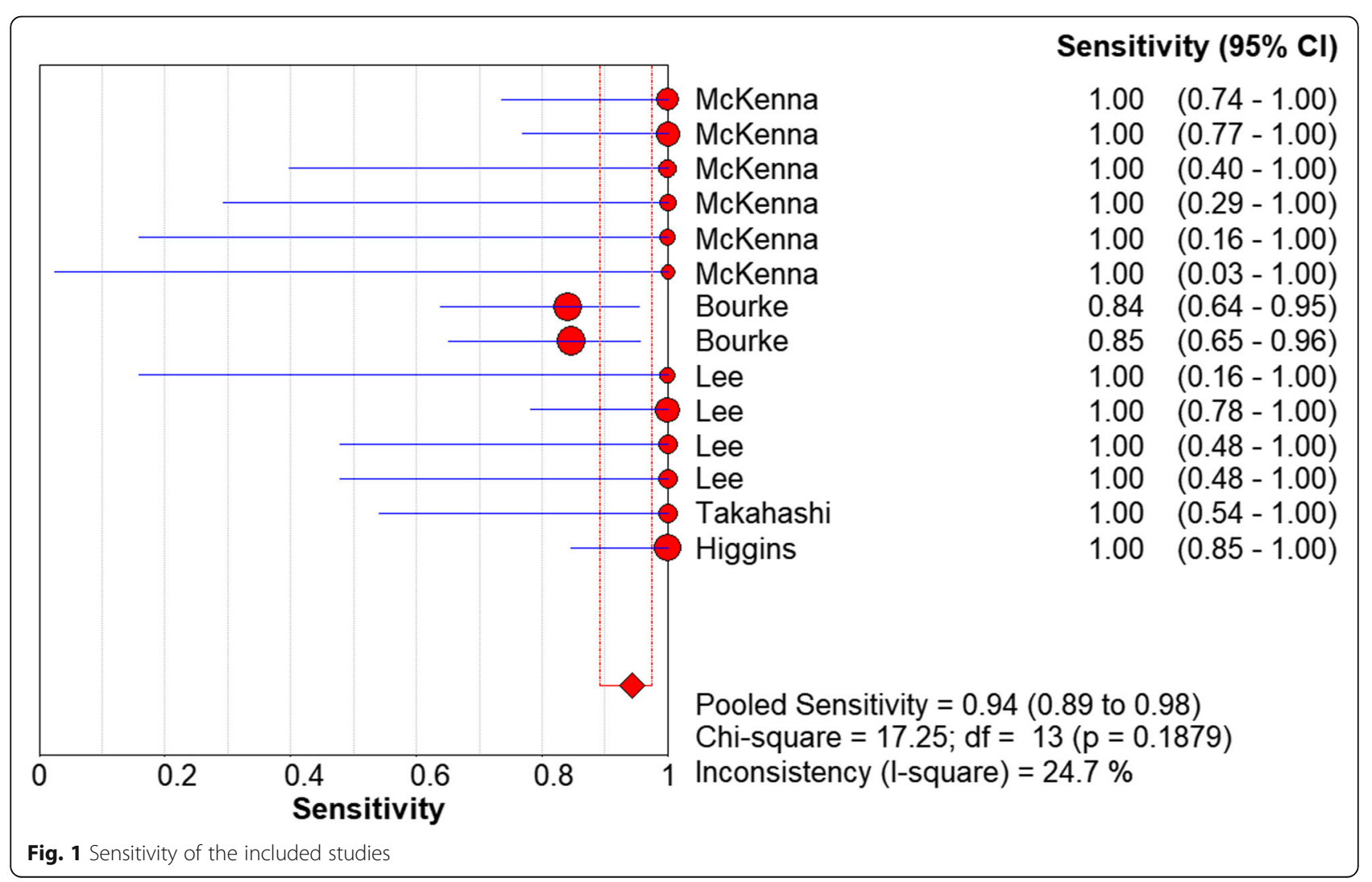




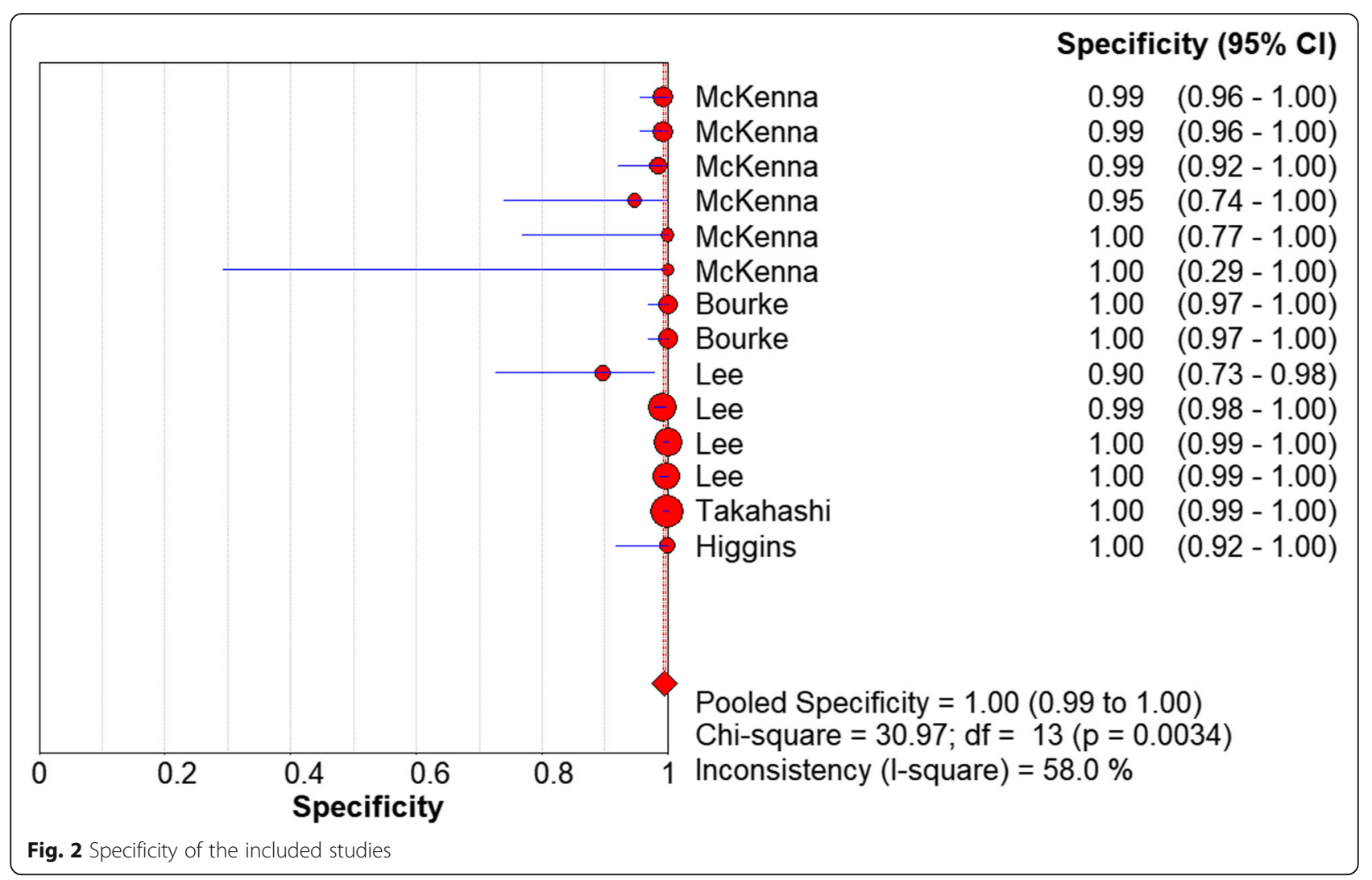

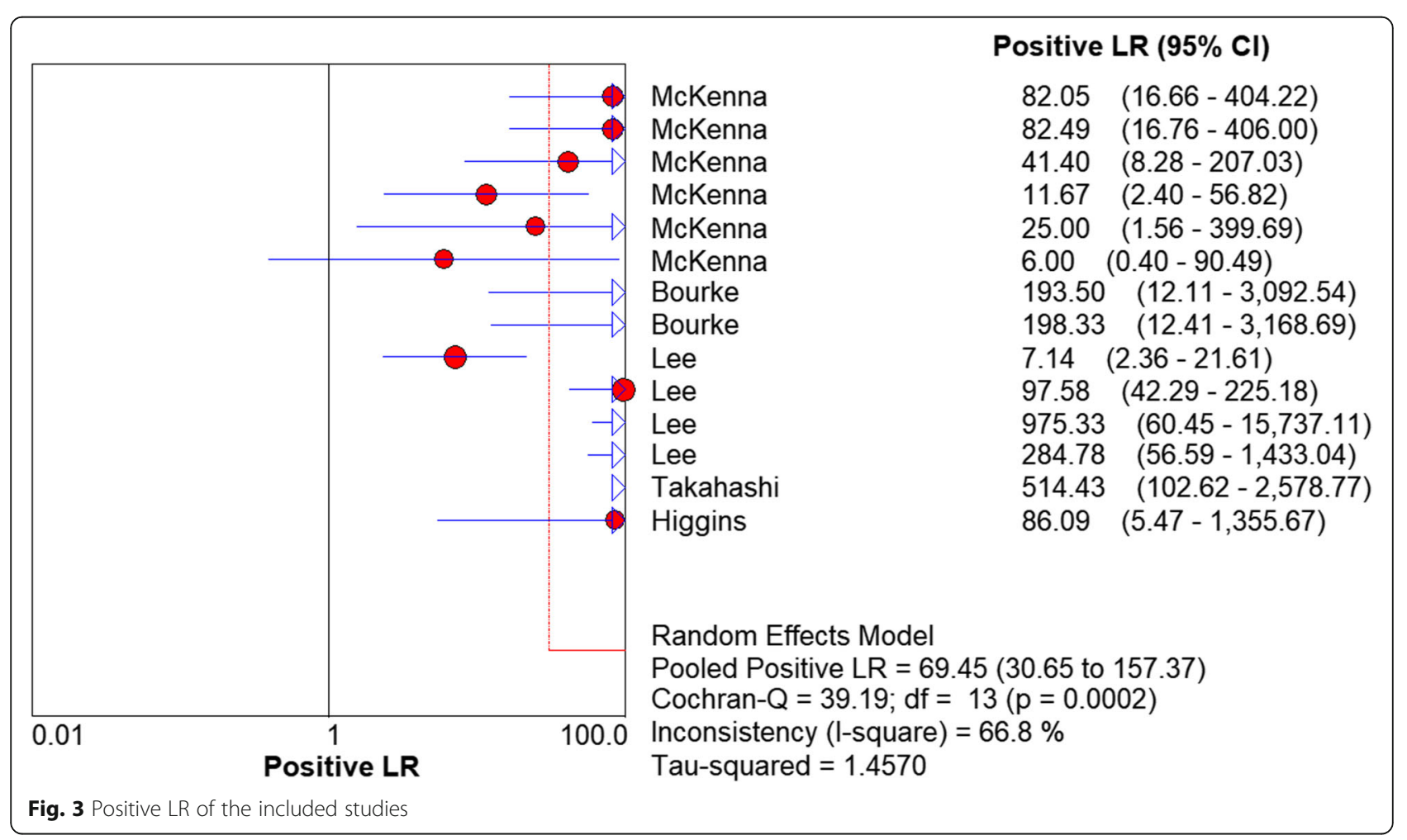




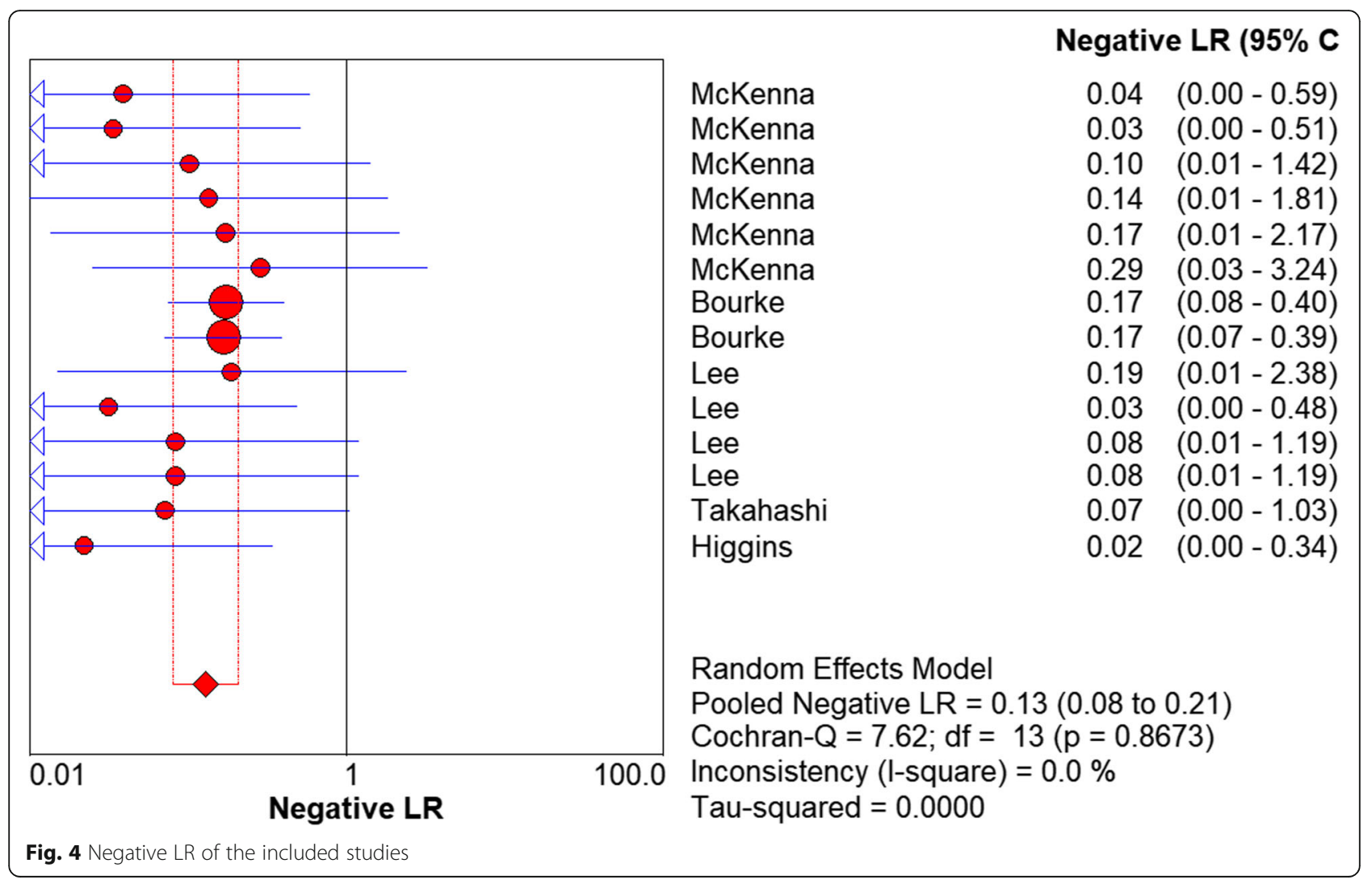

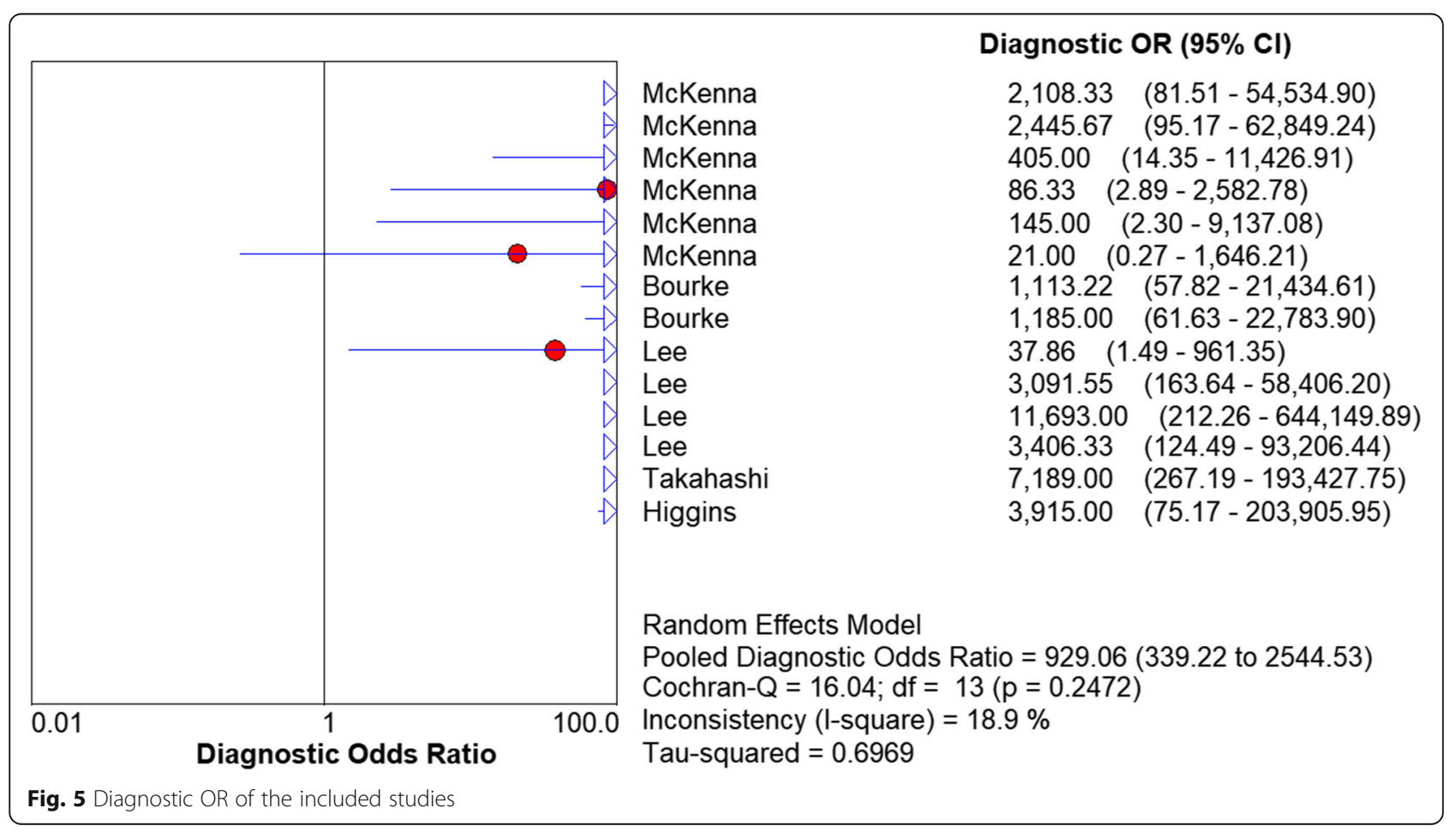




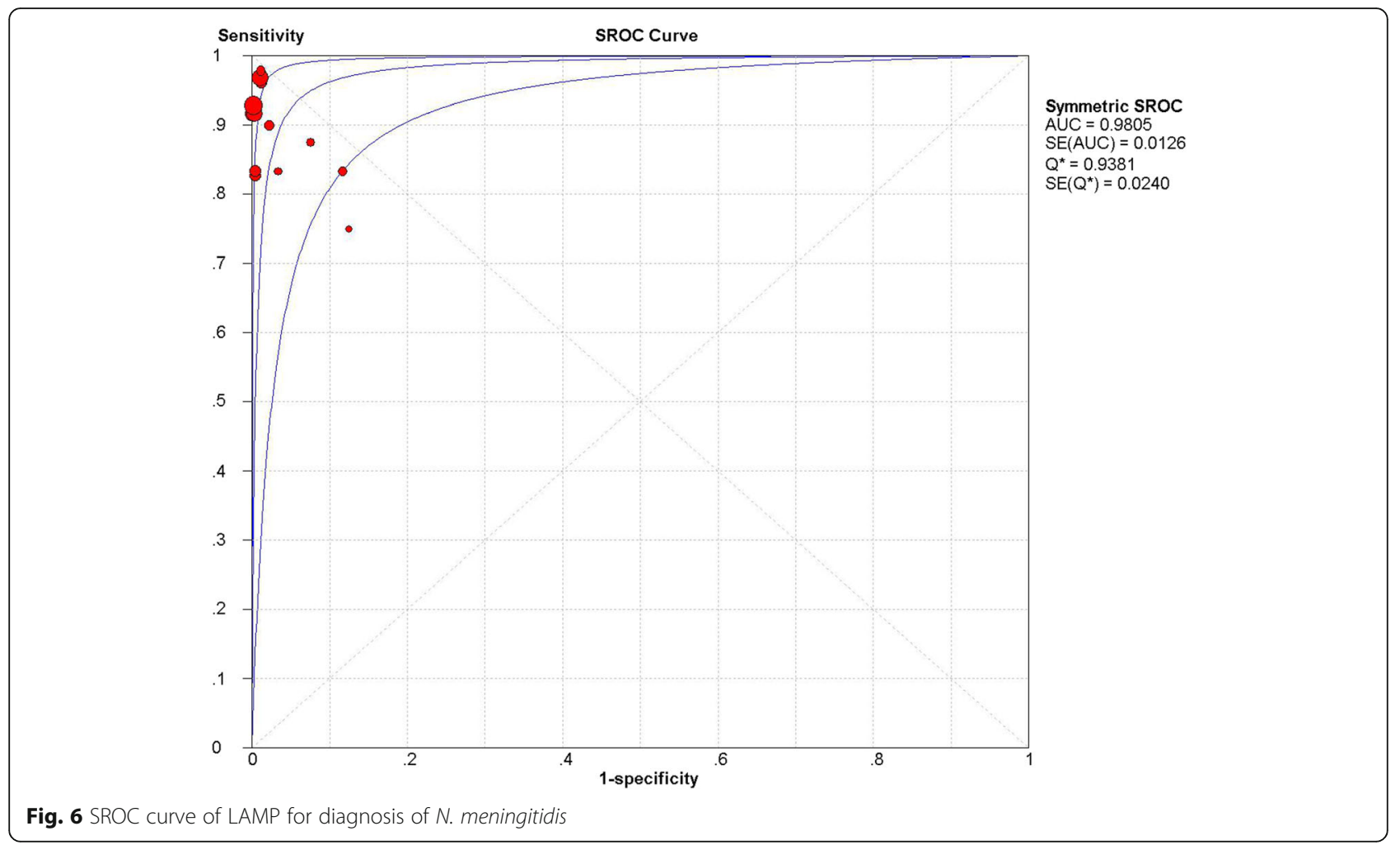

reference standards indicated an uncertain risk of bias in 4 studies and low risk of bias in 9 studies. Analysis of applicability concerns indicated low concerns in all three categories.

\section{Publication bias}

Deek's funnel plot (Fig. 9) showed that publication bias was not significant $(P=0.07)$. A bivariate box plot of sensitivity and specificity showed that there was no significant heterogeneity among the included studies (Fig. 10). The post-test probability nomogram showed that for samples with a predicted probability of $50 \%$, the post-test probability of a positive result was $100 \%$, and the post-test probability of a negative result was $1 \%$ (Fig. 11).

\section{Discussion}

Bacterial meningitis caused by Neisseria meningitidis has high global incidence and mortality rates, and is an especially severe problem in undeveloped regions. At present, routine laboratory diagnosis of bacterial meningitis employs bacterial culture and Gram staining, but this method is limited because it has a low sensitivity and is very time-

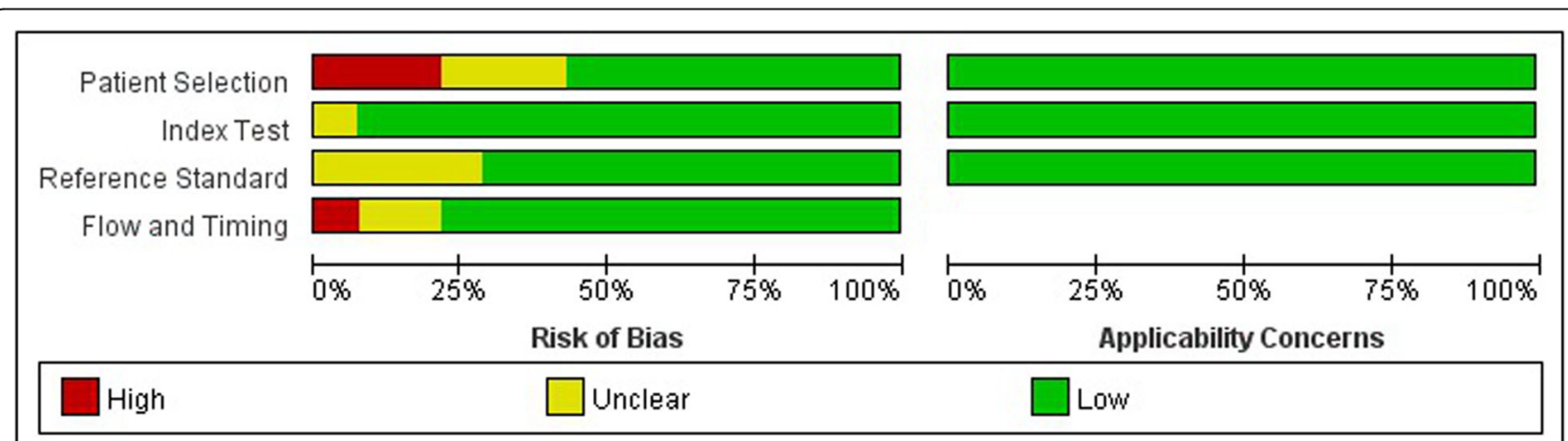

Fig. 7 Overall quality assessment of the included studies 


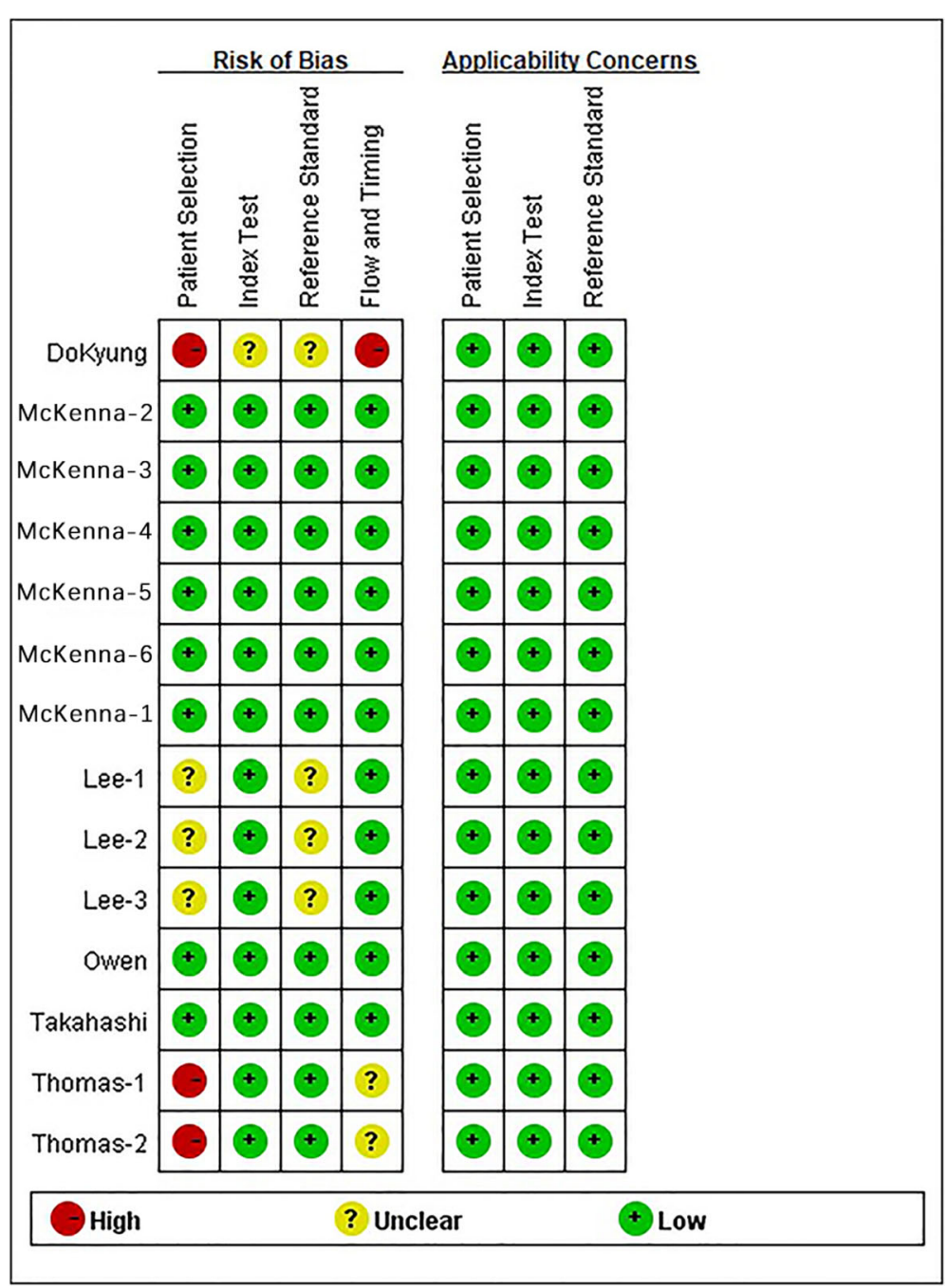

Fig. 8 Quality assessment of the individual studies

consumin $g[18,19]$. PCR is a widely accepted laboratory test for diagnosis of bacterial meningitis, and many studies have confirmed its high sensitivity and specificity. Unfortunately, the current PCR applications used to detect bacterial meningitis are too complicated and expensive for clinicians in undeveloped region s[19, 20]. Thus, existing PCR tests are difficult to apply in areas with the highest incidences of bacterial meningitis. A rapid, low-cost, easy to use, and highly sensitive technique is needed for these at-risk populations.

LAMP is a simple technique developed in recent years that uses 4 specific primers in 6 regions of the target gene and does not require a thermocycler. Under the action of a strand displacement DNA polymerase (Bst DNA polymerase), genes are amplified at a constant temperature of 60 to $65^{\circ} \mathrm{C}$, and amplification can be $10^{9}$-fold to $10^{10}$-fold in 15 to $60 \mathrm{~min}$. Higgins et al .[17] used Tth endonuclease
IV and a unique LAMP primer/probe to develop novel real-time multiplex LAMP technology, TEC-LAMP. Its main advantages are that it is simple, fast, and inexpensiv $\mathrm{e}[12,21]$.

We performed a pooled-analysis of 14 independent studies reported in 6 publications. These studies were performed in diverse geographic regions and used a variety of different samples. Thus, the study of McKenna et al .[13] was from Ireland and had 139 throat swab samples, 141 plasma samples, 72 blood samples, 22 cerebrospinal fluid samples, 16 respiratory secretion samples, and 4 stool samples. The study of Bourke et al .[14] was from the United Kingdom and had 141 nasopharyngeal swab samples and 144 blood samples. The study of Lee et al .[15] was from South Korea and examined 31 cerebrospinal fluid samples. The study of Lee et al .[16] was also from South Korea and examined 1574 cerebrospinal fluid samples (568 from Vietnam, 536 from China, and 470 from 


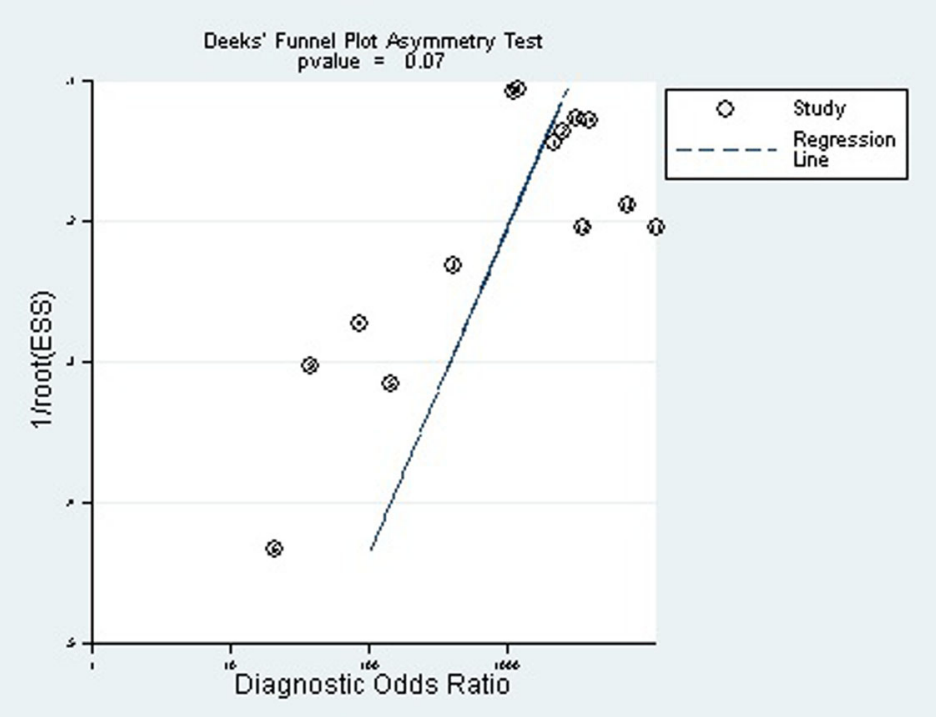

Fig. 9 Deek's funnel plot asymmetry test

South Korea). The study of Takahashi et al .[1] was from Japan and examined 836 throat swab samples. The study of Higgins et al .[17] was from Ireland and examined 65 blood samples. The study of Lee et al .[14] used culturing as the reference standard, and all the other studies used PCR as the reference standard. Our analysis results indicated that LAMP had an overall sensitivity of $94 \%$ ( $95 \% \mathrm{CI}=0.89$ to 0.98$)$ and an overall specificity of $100 \%$ ( $95 \% \mathrm{CI}=0.99$ to 1.00$)$ for the detection of $N$. meningitidis, and that the accuracy was high regardless of the sample type and geographic origin of the patients. Therefore, LAMP is highly suitable for the rapid, accurate, and inexpensive clinical detection of $N$. meningitidis in economically impoverished areas. Further prospective studies are needed to verify the safety and most suitable samples for the clinical use of LAMP.

\section{Conclusions}

In conclusion, our research showed that the LAMP assay had high sensitivity and specificity in detecting bacterial

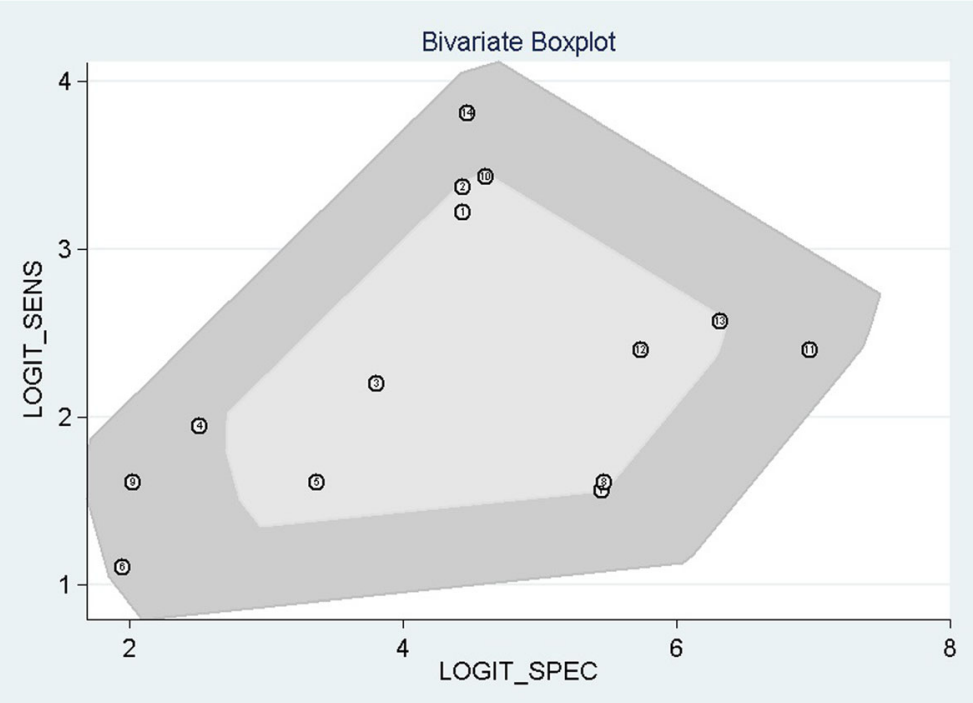

Fig. 10 Bivariate boxplot of the relationship of sensitivity and specificity 


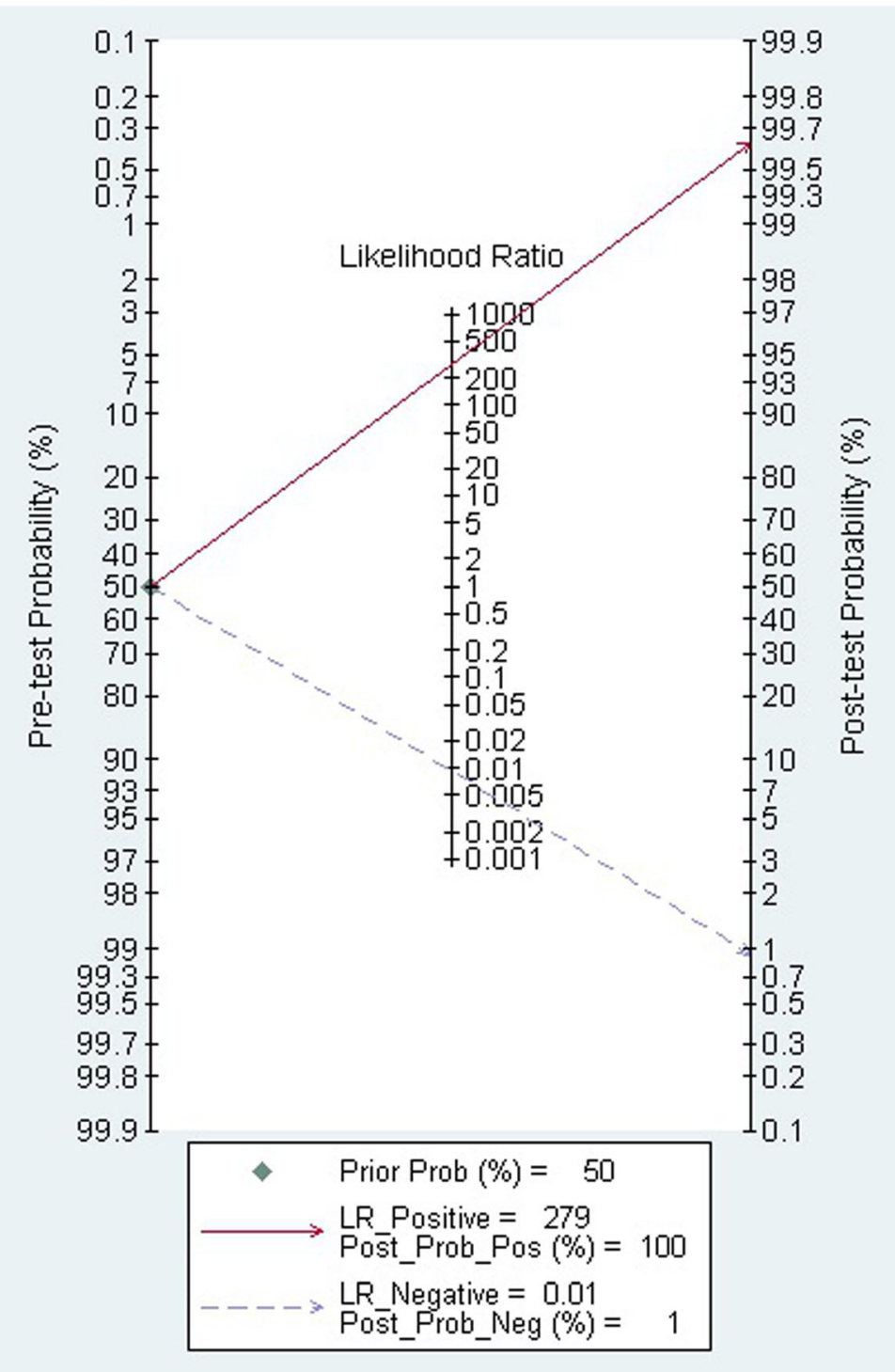

Fig. 11 Fagan nomogram of disease probabilities based on Bayes' theorem

meningitis due to $N$. meningitidis. This assay thus provides reliable information for clinical laboratory tests, and is suitable for detecting bacterial meningitis. However, further research is needed to verify the clinical feasibility and accuracy of this test in the impoverished areas where it is most needed.

\section{Supplementary information}

Supplementary information accompanies this paper at https://doi.org/10. 1186/s12879-020-05250-w.

Additional file 1: Figure S1. Identification and selection of the included studies.

\section{Abbreviations}

LAMP: Loop-mediated isothermal amplification; SROC: Summary receiver operating characteristic; PCR: Polymerase Chain Reaction; ELISA: Enzyme Linked Immuno Sorbent Assay; TP: True positivity; FP: False positivity; FN: False negativity; TN: True negativity; PLR: Positive likelihood ratio; NLR: Negative likelihood ratio; DOR: Diagnostic odds ratio

\section{Acknowledgements}

We express our sincere gratitude to all members of our study team for their cooperation and to the original authors of the studies included for their wonderful work and valuable participation.

Authors' contributions

XGG, FSJ, THK, XYC, CYZ, XTA, PZY,OS, LQ, LXY and LZX participated in the design of the project, formulation of the search strategy, and determination of inclusion of exclusion criteria. All authors participated in the literature search, data extraction and processing, and quality evaluation. XGG has made substantial contributions to the conception and design of the work. 
FSJ created the figures and table. FSJ and THK wrote the manuscript. All researchers read and approved the final version of the manuscript.

\section{Funding}

This work was supported in part by The Third Affiliated Hospital of

Guangzhou Medical University, number 2019A013.

\section{Availability of data and materials}

All data generated or analyzed during this study are included in this published article and its supplementary information files.

Ethics approval and consent to participate

Not applicable.

\section{Consent for publication}

Not applicable.

\section{Competing interests}

There is no competing interests in research.

\section{Author details}

'Department of Clinical Laboratory Medicine, the Third Affiliated Hospital of Guangzhou Medical University, Guangzhou 510150, China. ${ }^{2}$ Department of Clinical Medicine, the Third Clinical School of Guangzhou Medical University, Guangzhou 511436, China. ${ }^{3}$ Department of infectious disease, The Fifth Affiliated Hospital of Guangzhou Medical University, Guangzhou, China. ${ }^{4}$ Pulmonary, Critical Care and Sleep Medicine, Yale School of Medicine, New Haven, USA. ${ }^{5}$ Department of Laboratory Medicine, The Affiliated Shunde Hospital of Guangzhou Medical University, Foshan, China. ${ }^{6}$ Department of respiratory, The third Affiliated Hospital of Guangzhou Medical University, Guangzhou, China. 'Key Laboratory for Major Obstetric Diseases of Guangdong Province, Guangzhou 510150, China. ${ }^{8}$ Key Laboratory of Reproduction and Genetics of Guangdong Higher Education Institutes, Guangzhou 510150, China.

Received: 5 August 2019 Accepted: 14 July 2020

Published online: 20 July 2020

\section{References}

1. Takahashi $\mathrm{H}$, et al. Meningococcal carriage rates in healthy individuals in Japan determined using loop-mediated isothermal amplification and oral throat wash specimens. J Infect Chemother. 2016;22(7):501-4.

2. Seki $M$, et al. Loop-mediated isothermal amplification methods for diagnosis of bacterial meningitis. Front Pediatr. 2018:6:57.

3. Harrison LH, Trotter CL, Ramsay ME. Global epidemiology of meningococcal disease. Vaccine. 2009;27:B51-63.

4. Borrow $\mathrm{R}$, et al. The global meningococcal initiative: global epidemiology, the impact of vaccines on meningococcal disease and the importance of herd protection. Expert Rev Vaccines. 2017;16(4):313-28.

5. Memish ZA, et al. Invasive meningococcal disease and travel. J Infect Public Health. 2010;3(4):143-51.

6. Dou M, et al. Multiplexed instrument-free meningitis diagnosis on a polymer/paper hybrid microfluidic biochip. Biosens Bioelectron. 2017;87: 865-73.

7. Mihret W, et al. Molecular studies of meningococcal and pneumococcal meningitis patients in Ethiopia. Innate Immun. 2019;25(3):158-67.

8. Booy $\mathrm{R}$, et al. Recent changes in the epidemiology of Neisseria meningitidis serogroup $\mathrm{W}$ across the world, current vaccination policy choices and possible future strategies. Hum Vaccines Immunother. 2019:15(2):470-80

9. Guiducci $S$, et al. Culture and real-time polymerase chain reaction sensitivity in the diagnosis of invasive meningococcal disease: does culture miss less severe cases? PLoS One. 2019;14(3):e0212922.

10. Safavieh $\mathrm{M}$, et al. Emerging loop-mediated isothermal amplification-based microchip and microdevice Technologies for Nucleic Acid Detection. ACS Biomaterials Sci Eng. 2016;2(3):278-94.

11. Dou M, et al. A paper/polymer hybrid CD-like microfluidic SpinChip integrated with DNA-functionalized graphene oxide nanosensors for multiplex qLAMP detection. Chem Commun. 2017:53(79):10886-9.

12. Francois $P$, et al. Robustness of a loop-mediated isothermal amplification reaction for diagnostic applications. FEMS Immunol Med Microbiol. 2011; $62(1): 41-8$.
13. McKenna JP, et al. Development and clinical validation of a loop-mediated isothermal amplification method for the rapid detection of Neisseria meningitidis. Diagn Microbiol Infect Dis. 2011;69(2):137-44.

14. Bourke TW, et al. Diagnostic accuracy of loop-mediated isothermal amplification as a near-patient test for meningococcal disease in children: an observational cohort study. Lancet Infect Dis. 2015;15(5):552-8.

15. Lee, D., et al. A novel loop-mediated isothermal amplification assay for serogroup identification of Neisseria meningitidis in cerebrospinal fluid. Front Microbiol, 2016. 6(JAN).

16. Lee D, et al. Clinical Evaluation of a Loop-Mediated Isothermal Amplification (LAMP) Assay for Rapid Detection of Neisseria meningitidis in Cerebrospinal Fluid (vol 10, e0122922, 2015). PLoS One. 2015;10(6).

17. Higgins $\mathrm{O}$, et al. Evaluation of an internally controlled multiplex Tth endonuclease cleavage loop-meditated isothermal amplification (TEC-LAMP) assay for the detection of bacterial meningitis pathogens. Int J Mol Sci. 2018;19(2).

18. KENNEDY WA, et al. Incidence of bacterial meningitis in Asia using enhanced CSF testing: polymerase chain reaction, latex agglutination and culture. Epidemiol Infect. 2007;135(07).

19. Huy NT, et al. Development of a single-tube loop-mediated isothermal amplification assay for detection of four pathogens of bacterial meningitis. FEMS Microbiol Lett. 2012;337(1):25-30.

20. Chiba N, et al. Rapid detection of eight causative pathogens for the diagnosis of bacterial meningitis by real-time PCR. J Infect Chemother. 2009; 15(2):92-8.

21. Dou M, et al. A versatile PDMS/paper hybrid microfluidic platform for sensitive infectious disease diagnosis. Anal Chem. 2014;86(15):7978-86.

\section{Publisher's Note}

Springer Nature remains neutral with regard to jurisdictional claims in published maps and institutional affiliations.
Ready to submit your research? Choose BMC and benefit from:

- fast, convenient online submission

- thorough peer review by experienced researchers in your field

- rapid publication on acceptance

- support for research data, including large and complex data types

- gold Open Access which fosters wider collaboration and increased citations

- maximum visibility for your research: over $100 \mathrm{M}$ website views per year

At $\mathrm{BMC}$, research is always in progress.

Learn more biomedcentral.com/submissions 